\title{
Late Embryogenesis Abundant (LEA) proteins in legumes
}

\section{Marina Battaglia and Alejandra A. Covarrubias*}

Departamento de Biología Molecular de Plantas, Instituto de Biotecnología, Universidad Nacional Autónoma de Mexico, Cuernavaca, Mexico

\section{Edited by:}

Georgina Hernandez, Universidad

Nacional Autonóma de México,

Mexico

Reviewed by:

Manuel Becana, Consejo Superior de Investigaciones Científicas, Spain Xiaoping Chen, Guangdong

Academy of Agricultural Sciences, China

*Correspondence:

Alejandra A. Covarrubias,

Departamento de Biología

Molecular de Plantas, Instituto de

Biotecnología, Universidad Nacional

Autónoma de México,

Av. Universidad 2001, Col. Chamilpa,

Apdo Postal 510-3, 62210

Cuernavaca, Morelos, Mexico

e-mail: crobles@ibt.unam.mx
Plants are exposed to different external conditions that affect growth, development, and productivity. Water deficit is one of these adverse conditions caused by drought, salinity, and extreme temperatures. Plants have developed different responses to prevent, ameliorate or repair the damage inflicted by these stressful environments. One of these responses is the activation of a set of genes encoding a group of hydrophilic proteins that typically accumulate to high levels during seed dehydration, at the last stage of embryogenesis, hence named Late Embryogenesis Abundant (LEA) proteins. LEA proteins also accumulate in response to water limitation in vegetative tissues, and have been classified in seven groups based on their amino acid sequence similarity and on the presence of distinctive conserved motifs. These proteins are widely distributed in the plant kingdom, from ferns to angiosperms, suggesting a relevant role in the plant response to this unfavorable environmental condition. In this review, we analyzed the LEA proteins from those legumes whose complete genomes have been sequenced such as Phaseolus vulgaris, Glycine max, Medicago truncatula, Lotus japonicus, Cajanus cajan, and Cicer arietinum. Considering their distinctive motifs, LEA proteins from the different groups were identified, and their sequence analysis allowed the recognition of novel legume specific motifs. Moreover, we compile their transcript accumulation patterns based on publicly available data. In spite of the limited information on these proteins in legumes, the analysis and data compiled here confirm the high correlation between their accumulation and water deficit, reinforcing their functional relevance under this detrimental conditions.

Keywords: legumes, common bean, soybean, Medicago, LEA proteins, water deficit, abiotic stress

\section{INTRODUCTION}

Plants are subjected to different stresses (abiotic and biotic) along their life cycles and have developed different responses to alleviate the damage, and survive to these impaired conditions (Bartels and Sunkar, 2005). Abiotic stresses such as drought, salinity, osmotic, cold, and freezing temperatures produce cellular water deficit, which leads to the accumulation of a group of highly hydrophilic proteins, named LEA proteins (for Late Embryogenesis Abundant) (for review Battaglia et al., 2008; BiesEtheve et al., 2008; Hundertmark and Hincha, 2008). These proteins are not only associated to water deficit caused by environmental changes but also to water limitation produced during plant development under optimal growth conditions, such as during development of seeds and pollen grains, or some stages of shoot and root development (Colmenero-Flores et al., 1999; Vicient et al., 2000; Sheoran et al., 2006). Some LEA proteins have also been found associated to vascular tissues, and also in meristematic regions (Cheng et al., 1996; Colmenero-Flores et al., 1999; Moreno-Fonseca and Covarrubias, 2001). Their high accumulation in embryos of dormant seeds and in mature pollen grains, both desiccation resistant structures able to withstand dehydration for long periods, led to propose a role for these

Abbreviations: Groups of LEA proteins: LEA1, LEA2, LEA3, LEA4, LEA6, and LEA7. proteins in plant tolerance to water scarcity [for review see Dure III et al. (1981), Battaglia et al. (2008), Bies-Etheve et al. (2008), Hundertmark and Hincha (2008)].

LEA proteins have been found widely distributed in the plant kingdom, from algae (Honjoh et al., 1995; Joh et al., 1995), moss (Saavedra et al., 2006), ferns (Raghavan and Kamalay, 1993), to angiosperms. Interestingly, LEA-like proteins also accumulate in anhydrobiotic invertebrates and in some bacterial species in response to water limitation (Stacy and Aalen, 1998; Browne et al., 2004; Goyal et al., 2005a; Kikawada et al., 2006; Wang et al., 2007; Hand et al., 2011; Clark et al., 2012).

Most LEA proteins identified to date belong to hydrophilins, a widely distributed group of proteins characterized by a high content of charged amino acid residues, as well as glycine or other small amino acids such as alanine, serine, or threonine. Moreover, most hydrophilins lack tryptophanes and cysteines (Garay-Arroyo et al., 2000). These physicochemical properties led to propose that hydrophilins are unstructured proteins in aqueous solutions (Dure, 1993; Garay-Arroyo et al., 2000), which has been shown for some LEA proteins [see for review Olvera-Carrillo et al. (2011)]. Analyses of LEA protein amino acid sequences recognize seven different groups, each one showing distinctive motifs (LEA1 - LEA7). In particular, for groups 2, 3, and 4 different protein variants have been found showing a particular organization of their corresponding motifs (Figure 1). Significant 


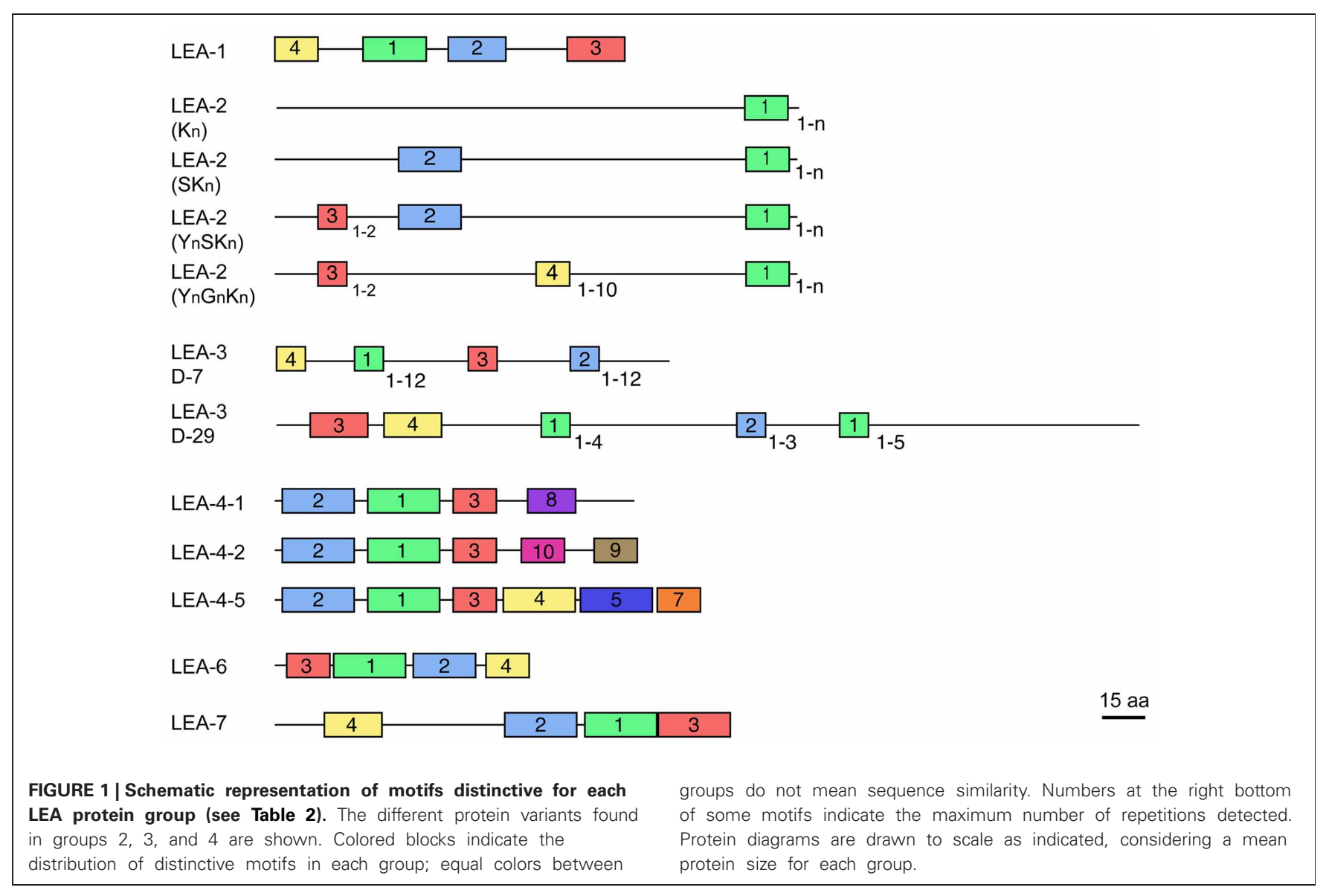

sequence similarity has not been detected between the seven LEA protein groups. Because of their more hydrophobic nature and higher structural order, group 5 LEA proteins are not classified as hydrophilins (Dure III et al., 1989; Battaglia et al., 2008). Given the high heterogeneity and little information on this group, it was not included in this review.

A variety of studies have shown a potential role for LEA proteins in stress tolerance. Their function in protein protection upon water deficit was demonstrated by in vitro experiments, where different hydrophilins, including LEA proteins from groups 2,3 , and 4 , were able to prevent the inactivation of enzymes such as LDH (lactate dehydrogenase) or MDH (malate dehydrogenase) upon different dehydration levels (Goyal et al., 2005b; Reyes et al., 2005, 2008). Similarly, protective properties were also detected during freeze-thaw in vitro assays (Hara et al., 1999; Reyes et al., 2008). Interestingly, in these latter assays, a particular protective role was detected for the K-segment, a LEA2 protein distinctive motif, and for a conserved region in LEA4 proteins (Reyes et al., 2008). Because in these assays, 1:1 ratios between LEA and target proteins were enough to provide protection, it was proposed that this protective activity is carried out by protein-protein interactions (Reyes et al., 2005; Olvera-Carrillo et al., 2011). More severe in vitro dehydration assays, using high LEA: target protein ratios, suggested that some LEA and LEAlike proteins are also able to avoid protein aggregation (Goyal et al., 2005b; Chakrabortee et al., 2007). In addition, some reports suggest that the presence of sugars can enhance LEA proteins protecting effect under dehydration (Black et al., 1999; Wolkers et al., 2001; Liu et al., 2010).

Membrane stabilization has been another function attributed to LEA proteins. Most evidence comes from in vitro experiments, where LEA2 proteins (dehydrins) were found associated to anionic phospholipid vesicles (Koag et al., 2003, 2009). Also, other assays suggested that dehydrin addition maintain the functional membrane structure under freezing temperatures (Ismail et al., 1999; Kosová et al., 2007). These data are in agreement with reports that have detected binding of dehydrins to membranes or phospholipids; however, all these results have been obtained from in vitro experiments or with isolated membranes. In contrast, no conclusive information is available regarding their membrane association by intracellular localization experiments using antibodies or fluorescent translational fusions, which however, have contributed to establish dehydrins location in cytosol, nuclei, chloroplast, protein and lipid bodies, mitochondria, and plasmodesmata [for review Rorat (2006)].

In the case of LEA proteins from other groups, most of them have been shown to be cytosolic; however, some have also been localized in chloroplast (Ndong et al., 2002), mitochondria (Grelet et al., 2005; Tolleter et al., 2010) and even in nucleus (Goday et al., 1994; Houde et al., 1995; Colmenero-Flores et al., 1999; Riera et al., 2004; Gai et al., 2011; Duan and Cai, 2012). The mitochondrial localization of pea (Pisum sativum) LEA3 
proteins correlates with their ability to protect some mitochondrial enzymes such as rhodanese and fumarase from inactivation induced by in vitro dehydration (Grelet et al., 2005). Their nuclei localization has also been associated with binding to DNA for some LEA2, LEA4, and LEA7 proteins (Colmenero-Flores et al., 1999; Maskin et al., 2007; Hara et al., 2009).

Additional roles have been suggested for these proteins such as ion sequestration, in particular, for LEA2 and LEA4 proteins, where histidine-containing motifs seem to be responsible of binding divalent cations (Hara et al., 2005). This is the case of soybean LEA4-5 proteins (GmPM1: Glyma19g32920.1 and GmPM9: Glyma03g30040.1), which bind $\mathrm{Fe}^{+3}, \mathrm{Ni}^{+2}, \mathrm{Cu}^{+2}$ and $\mathrm{Zn}^{+2}$ (Liu et al., 2011). In vitro experiments using a citrus dehydrin, or GmPM1 and GmPM9 indicate that these proteins reduce the levels of $\mathrm{OH}^{\cdot}$ radical; hence, suggesting an oxidant scavenger activity associated to an increased ion concentration (Hara et al., 2005; Liu et al., 2011).

Over-expression experiments in different organisms have also contributed to support a role of LEA proteins in tolerance to water scarcity. For instance, expression of soybean PM2 protein (LEA3) conferred Escherichia coli with the ability to grow under high salt or upon extreme temperature treatments (Liu and Zheng, 2005; Liu et al., 2010). Recently, it has been shown that a LEA3-like protein from the brine shrimp Artemia franciscana increases cellular viability after desiccation and hyperosmotic stress of Drosophila melanogaster cells (Marunde et al., 2013). In plants, a number of reports indicate that over-expression of LEA proteins from various groups confers tolerance to a variety of water deficit treatments (Puhakainen et al., 2004; Eriksson and Harryson, 2011; Duan and Cai, 2012). More significantly, it has been shown that the deficiency of one or two of the three LEA4 proteins from Arabidopsis thaliana is enough to cause water deficit susceptibility (Olvera-Carrillo et al., 2010), showing their relevance in the plant adaptive response to this stress condition. This role is also endorsed for LEA2 proteins as indicated by the co-segregation of a dehydrin gene with chilling tolerance during cowpea (Vigna unguiculata L.) seedling emergence (Ismail et al., 1999).

Even though there is very limited information regarding the participation of LEA proteins in the plant response to biotic stress, it is known that over-expression of group 2 LEA proteins from Arabidopsis affects the expression of genes related to plant defense responses (e.g., pathogenesis-related proteins, PR) (Hanin et al., 2011); also, it has been reported that a group 3 LEA protein from maize is up-regulated in response to bacterial infection and that its over-expression in tobacco plants improves tolerance to Pseudomonas syringae (Liu et al., 2013). In addition, the over-expression in Escherichia coli of groups 2 and 4 LEA proteins causes bacterial growth inhibition (Campos et al., 2006; Hanin et al., 2011). These observations have led to the speculation that the antibacterial activity of some dehydrins may be part of a defense mechanism against opportunistic bacterial infections commonly occurring during water deficit conditions, a hypothesis awaiting exploration.

As it has been described above, the responsiveness of the different LEA genes to water deficit is common, however, some of them have also been found to be responsive to other stressful conditions such as ion toxicity, oxidation, or high temperatures, and in some cases a correlation has been found with the ability of LEA proteins to bind different cations, including $\mathrm{Fe}^{+3}, \mathrm{Ni}^{+2}$, $\mathrm{Cu}^{+2}$, or to confer tolerance to these stress conditions [for review Hanin et al. (2011)]. Even though these observations may be difficult to integrate, the wide variety of functions that have been attributed to these proteins could be related to their selection throughout evolution not only by low water availability but also by other intrinsically associated conditions; that would be the case of high temperatures or the generation of high ion concentrations and reactive oxygen species, among other effects. In addition, the flexibility of their structure should also be considered, as for many IUPs it has been associated to multifunctionality (Hara, 2010; Liu et al., 2010, 2013; Olvera-Carrillo et al., 2011; Dominguez et al., 2013), an appealing characteristic that has been poorly explored in these proteins.

\section{LEA PROTEINS IN LEGUMES}

To gain insight into the conservation patterns of the different distinctive motifs identified in LEA proteins, in this work we analyzed the different legume LEA proteins sequences using the information available in the legume genome sequence databases (Phaseolus vulgaris: http://www.phytozome.net/search.php?org=Org_Pvulgaris_v1.0; Glycine max: http://www.phytozome.net/search.php?org=Org Gmax_v1.1; Medicago truncatula: http://medicago.jcvi.org/ cgi-bin/medicago/overview.cgi; Lotus japonicus: http://www. kazusa.or.jp/lotus/), Cajanus cajan (http://cajca.comparativelegumes.org/) (Varshney et al., 2012) and Cicer arietinum (http://cicar.comparative-legumes.org/) (Varshney et al., 2013) (see Table 1). For this, protein sequences from each group were compared and the motifs described for Viridiplantae were analyzed (see Table 2 and Figure 1). In addition to the strong similarity between those motifs previously identified (Battaglia et al., 2008), a new Gly-rich region was found in some legume LEA2 proteins. The most conserved motif in this group is a repetitive 15-mer motif, EKKGIMDKIKEKLPG, called K-segment because of its richness in lysine $(\mathrm{K})$ residues. Additional common motifs are the Y- and S-segments. When present, the S-segment usually precedes the K-segment (Campbell and Close, 1997). The new glycine-rich region detected shows a repetitive large motif represented by the sequence [YF]T[GD]DT[GN][RK]QH[GD]T. This sequence is present up to 10-times in a protein, as in the case of the polypeptide encoded by the Phvul004G158800.1 gene from $P$. vulgaris. Two dehydrins with Gly-rich motifs have been described. One from Vigna radiata, VrDhn1, a novel $\mathrm{Y} 2 \mathrm{~K}$ dehydrin detected in seeds and induced by various abiotic stresses or ABA treatment (Lin et al., 2012), and Mat1 (Glyma07g10030.1) from soybean, which has a homolog called Mat9 (Glyma09g31740.2), induced upon severe water deficit (Whitsitt et al., 1997; Momma et al., 2003). This motif was only detected in LEA2 proteins from Phaseoloid species, which include several legumes more adapted to tropical climates. Because this group is considered one of the youngest groups among the three legume sub-families (Gepts et al., 2005), it can be suggested that this motif is of recent appearance. It is worth mentioning that this motif and its repetitions (4-10X) always were found to be present between segments- $\mathrm{Y}$ and $-\mathrm{K}$, which suggests a common ancestor for this type of dehydrins. 


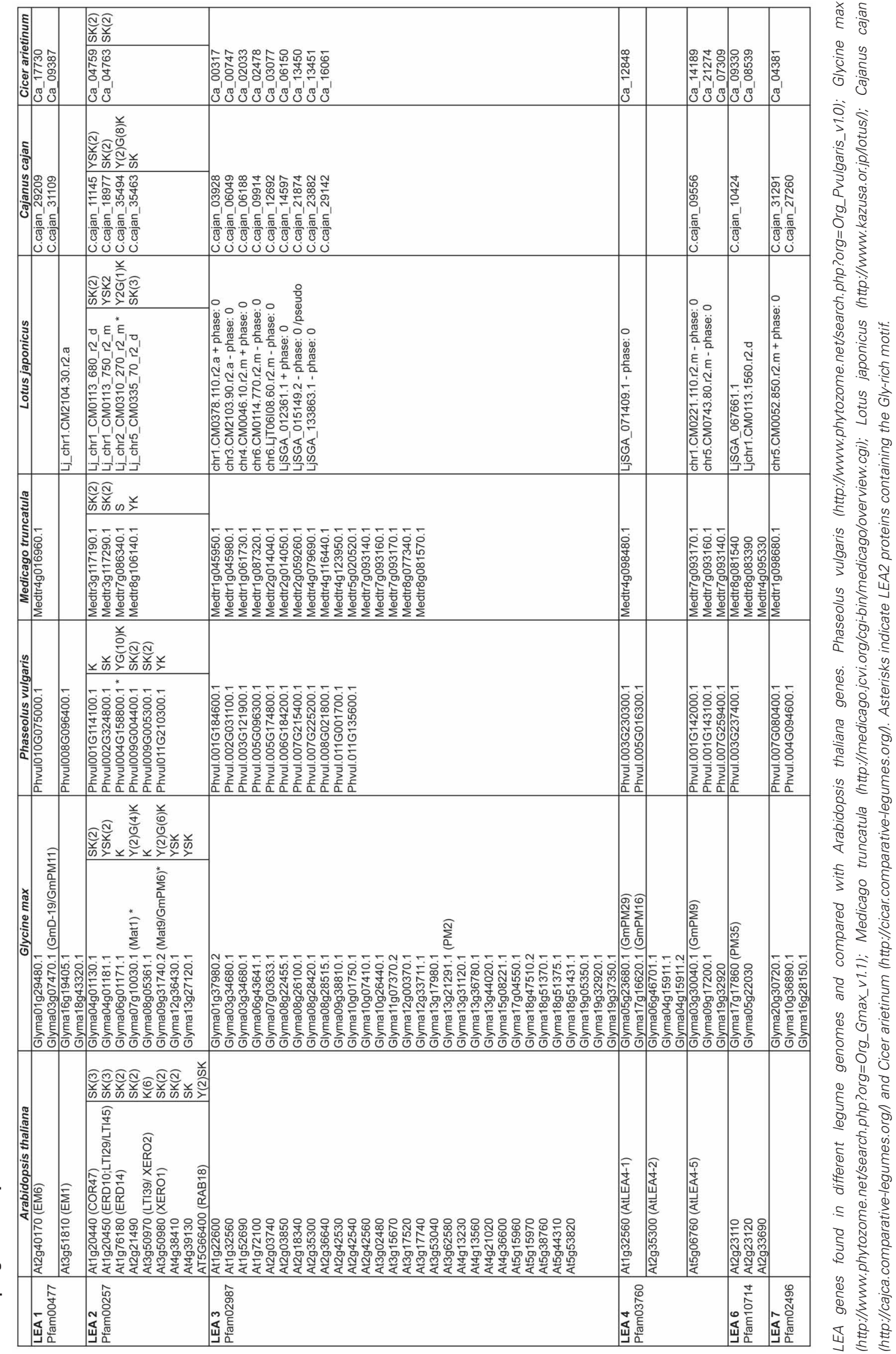


Table 2 | Distinctive motifs in the different groups of legume LEA proteins.

\begin{tabular}{ll}
\hline & Representative motifs \\
\hline & Motif $\mathbf{1}$ \\
\hline LEA1 & ETVVPGGTGGKSLEAQE[HN]LAEG \\
LEA2 & EKKG[IF][LM][ED]KIKEK[LI]PG \\
LEA3 D-7 & [TA]A[EQ]AAK[DN]K[TA][AQ][EQ] \\
LEA3 D-29 & YA[GSN]D[AT][AT][QD][KA][TA]K[DKE] \\
LEA4* & R[DT][PK]E[EQ]K[EV][IM]A[TH][EQ][KR][KAR][EK]AKE[AN] \\
& {$[$ QE]A[EK][ML][DE][KL][HQ] } \\
LEA6 & SPY[VL]KYKDLEDYKRQ[GA]YG[TA]QGHQ[EQ]P \\
LEA7 & DPE[HN][AE]H[RK]HKIEEE[VI]AAAAAVG[AS]GGF \\
\hline
\end{tabular}

\begin{tabular}{ll}
\hline & Motif $\mathbf{2}$ \\
\hline LEA1 & GQTRK[EQ]QLG[ST]EGY[HQ]EMGRKG \\
LEA2 & LL[EG]KL[HR]RS[DN]SSSSSSS[ED][ED][ED]GE \\
LEA3 D-7 & A[AS][QE]K[AT]K[ED]YA[GD][DY] \\
LEA3 D-29 & [YT][AK]D[DY][AT]AOK[TSA]K[DE] \\
LEA4* & KE[TK][AL][AKS]N[IM][GA][AS][SA]AK[AES][GK]M[ED][KI][TY] \\
& KA[TK][IV][QD]EK \\
LEA6 & K[PT]GRG[AGP]GAT[ED]A[PA]T[LP]SG[AD][AN]VSS \\
LEA7 & [ED]VDYKKEEKHHK[HK]LEHLGELGA[AT]AA \\
\hline
\end{tabular}

\begin{tabular}{ll}
\hline & Motif 3 \\
\hline LEA1 & M[DE][KE]SGGERA[AE][ER]EGI[DE]IDESKF \\
LEA2 & D[EQA][YH]GN[PV][VI][KH][LA]T \\
LEA3 D-7 & E[GATV]KD[KAT][TA][MV]EK[AL]G \\
LEA3 D-29 & [VF][LFT][VI][ILMV][AFL][LMV][MFV][LV][FLT][ATV][MAI][NS] \\
& [CV][NKRY][CS][TS][ST]V[GD]H[MI]P \\
LEA4* & [AE]A[RK][EA][HR][NH]AA[AE]K[QL]S[AH] \\
LEA6 & [ERS][ES]S[GK][KE][GS][SN][IT]EGLP[FM]ED \\
LEA7 & FHEHHEKKEAK[EK][EQ][DE]EE[AS]H[GS][KE]K[HR]HH[LF] \\
\hline
\end{tabular}

\begin{tabular}{ll}
\hline & Motif $\mathbf{4}$ \\
\hline LEA1 & MAS[KQ]Q[QA]N[RK][EQ]ELDE[RK]A \\
LEA2 & [YF]T[GD]DT[GN][RK]QH[GD]T \\
LEA3 D-7 & M[AD]S[QH]N[QM][SN]Y[QN]AG \\
LEA3 D-29 & EG[HR]DF[QE][ED] [AS V] KAKT[TM] [Q E]TAN KA[M S] \\
& [E D]T \\
LEA4* & - \\
LEA6 & [FQ]NAA[TD]DA[IV][NH]SK[GE][VA][PS] \\
LEA7 & HHKDE[DE][NT][KR]PIIT][DE]T[ED]TGY[DN][NK]TSY \\
\hline
\end{tabular}

\begin{tabular}{ll}
\hline & LEA4* (Subgroups) \\
\hline LEA4-1 & 8: NOSHYGLHHGHN[NP]PILP] \\
LEA4-2 & 9: D[PH]A[TM][VL]E[IA][TA]NGPASTI \\
& 10: VEKAG[RK]IAEKEISKH \\
LEA4-5 & 4: [HY]G[HQ][PH][MT]G[ALT]HQ[TM]SA[ML]PGHGTGQPTGHV \\
& 5: [HQ]PIIL]G[TA][NV][PR][GM]PG[GAT]T[AYT][PT][AST] \\
& [HY][PN][LT][GR][AG][GN][GP]NPINP] \\
& 7: [AD][TY]GY[GT]TGG[TG]Y[NKT]
\end{tabular}

LEA protein motifs were discovered using MEME algorithm (4.9.0 version), with an optimum width between 6-25 amino acids, selecting a motif distribution of 0-1 or any number of repetitions (Bailey et al., 2009). The asterisk after LEA4 indicates that for LEA4 proteins, the specific motifs for each sub-group are shown in a separate block. Colors in top bars for every block correspond to those used in Figure 2 for each motif.

\section{STRUCTURE}

The LEA protein amino-acid composition predicted structural disorder along their sequence (Dure, 1993; Garay-Arroyo et al., 2000), and thus inscribed them as intrinsically unstructured proteins (IUPs). Structural analyses using circular dichroism (CD) and Fourier transform infrared (FTIR) spectroscopy have shown that this is the case, at least, for some purified recombinant LEA proteins in aqueous solutions (Lisse et al., 1996; Thalhammer et al., 2010). For legume LEA proteins, this has been shown for LEA1 proteins such as GmD19 (Glyma03g07470.1), GmPM11 (Glyma03g07470), LEA2 as GmPM6 (Glyma09g31740.1-LEA2), LEA3 like GmPM30 (Glyma13g44020.1) and LEA4 (Glyma17g16620), where also a potential to attain a more ordered structure by using $\alpha$-helical inducer agents (i.e., TFE) was observed (Soulages et al., 2002, 2003; Shih et al., 2004, 2010, 2012). In addition, conformational changes could be induced by slow or fast drying that lead to the formation of different $\alpha$-helical proportions, depending on the protein (Shih et al., 2004, 2012). These results support the idea that unstructured LEA proteins are rather flexible proteins, able to adapt their conformation to the variable cellular environment as that produced by low water availability. It has been proposed that these conformational changes may facilitate interactions between LEA proteins and other macromolecules, such as other proteins, membranes and/or nucleic acids (Boudet et al., 2006; Zhang et al., 2006; Maskin et al., 2007; Battaglia et al., 2008; Hundertmark et al., 2011, 2012; Olvera-Carrillo et al., 2011; Popova et al., 2011).

\section{TRANSCRIPT AND PROTEIN EXPRESSION PATTERNS}

LEA proteins have been detected in different stages of plant development in response to different water deficit conditions (salinity, freezing, and drought). In legumes, this has been reported for common bean, soybean or Medicago truncatula seedlings (Blackman et al., 1991; Hsing et al., 1995; Colmenero-Flores et al., 1997; Boudet et al., 2006; Aghaei et al., 2009; Le et al., 2012). As expected, their accumulation during late embryogenesis has also been documented in M. truncatula (Kalaiselvi and Manickam, 1999; Chatelain et al., 2012), G. $\max$ (Shih et al., 2004), Arachis hypogaea (Su et al., 2011) and P. vulgaris (Colmenero-Flores et al., 1997). Although very little information exists regarding the regulation of the expression of $L E A$ genes, for some of them it is known that $\mathrm{ABA}$ induced their transcript accumulation (Colmenero-Flores et al., 1997; Bai et al., 2012).

To obtain more information on the accumulation patterns for legume LEA genes, we analyzed public microarrays and RNA-sequencing databases (M. truncatula:http://mtgea.noble.org/v2/blast_search_form.php; G. max: http://soykb.org/; Lotus japonicus: http://cgi-www.cs.au.dk/ cgi-compbio/Niels/index.cgi?page $=$ i). These data indicate that most legume LEA transcripts and proteins have similar accumulation patterns as those found for LEA proteins from other plant species. M. truncatula microarrays showed that water deficit conditions imposed with $\mathrm{NaCl}$ treatments $(200 \mathrm{mM})$ induce the accumulation of transcripts from LEA genes of groups 2, $3,4,6$, and 7. LEA1 transcripts were mostly detected during late embryogenesis as in Arabidopsis (Manfre et al., 2006, 2009), cotton (Dure III et al., 1989), and wheat (Litts et al., 1987, 1991). 
M. truncatula proteome analysis showed that MtEm6 protein is particularly present in newly emerged root tips (Zhang et al., 2006), as well as its transcript, as showed by microarray data.

\section{THEIR PRESENCE IN MERISTEMATIC REGIONS AND ROOT HAIRS}

Interestingly, scrutiny of soybean microarray information showed that $L E A$ transcripts from different groups accumulate in root tips and apical meristems, however, this just applies to some of them. For example, Glyma03g07470.1 (LEA1) was only detected in root tips, as did Glyma04g01130.1 (LEA2), and Glyma16g28150.1 (LEA7). All LEA3 and LEA4 transcripts accumulate in root tips except Glyma10g26440.1 (LEA3), Glyma17g04550.1 (LEA3), and Glyma09g17200.1 (LEA4). Accumulation in apical meristems was detected for Glyma04g01130.1 (LEA2), Glyma17g16620.1 (LEA3), and Glyma13g44020.1 (LEA3), all LEA 4 except Glyma05g23680.1, and all LEA7 transcripts. While soybean LEA6 transcripts were not detected in meristems, the transcript and protein for its homologous gene accumulate in vegetative and root meristems from principal and lateral roots of P. vulgaris (Colmenero-Flores et al., 1999; Moreno-Fonseca and Covarrubias, 2001). These observations strongly support the idea that LEA proteins could protect stem cells niches from changes in environmental water availability preventing and/or avoiding damage to these cells vital for plant survival.
A group of root epidermal cells, the atrichoblasts, has a specific cell fate to develop and differentiate as root hairs; they are formed by one tubular cell with polar growth (Cárdenas, 2009). Root hairs play a crucial role in plants, because water and nutrient uptake from soil occur principally through them. In legumes, root hairs have an additional role, they are the cells that interact, recognize and constitute the entrance of their symbiont, the rhizobial bacteria (Goormachtig et al., 2004). In these cells, LEA proteins have been detected in non-legumes growing under optimal irrigation conditions, as shown for AtEm6, a LEA1 gene (Vicient et al., 2000). Legume LEA transcript accumulation in root hairs was analyzed from a public database generated by comparing soybean transcriptome sequences obtained from free-or B. japonicum-inoculated root hairs (Libault et al., 2010a). Interestingly, LEA transcripts from all groups were found to accumulate in root hairs of non-infected plants (Figure 2), such as LEA2 (Glyma04g01130.1, Glyma04g01180.1, Glyma07g10030.1, and Glyma09g31740.1), LEA3, LEA4 (all), LEA6 (Glyma17g17860.1), and LEA7 (Glyma16g28150.1); however, none of them showed higher accumulation by rhizobia infection. Analysis of a proteome from isolated root hair cells identified the accumulation of only three LEA proteins: Glyma13g31120; Glyma03g34680 (LEA3), and Glyma04g01130.1 (LEA2), most probably due to the limitations inherent to the

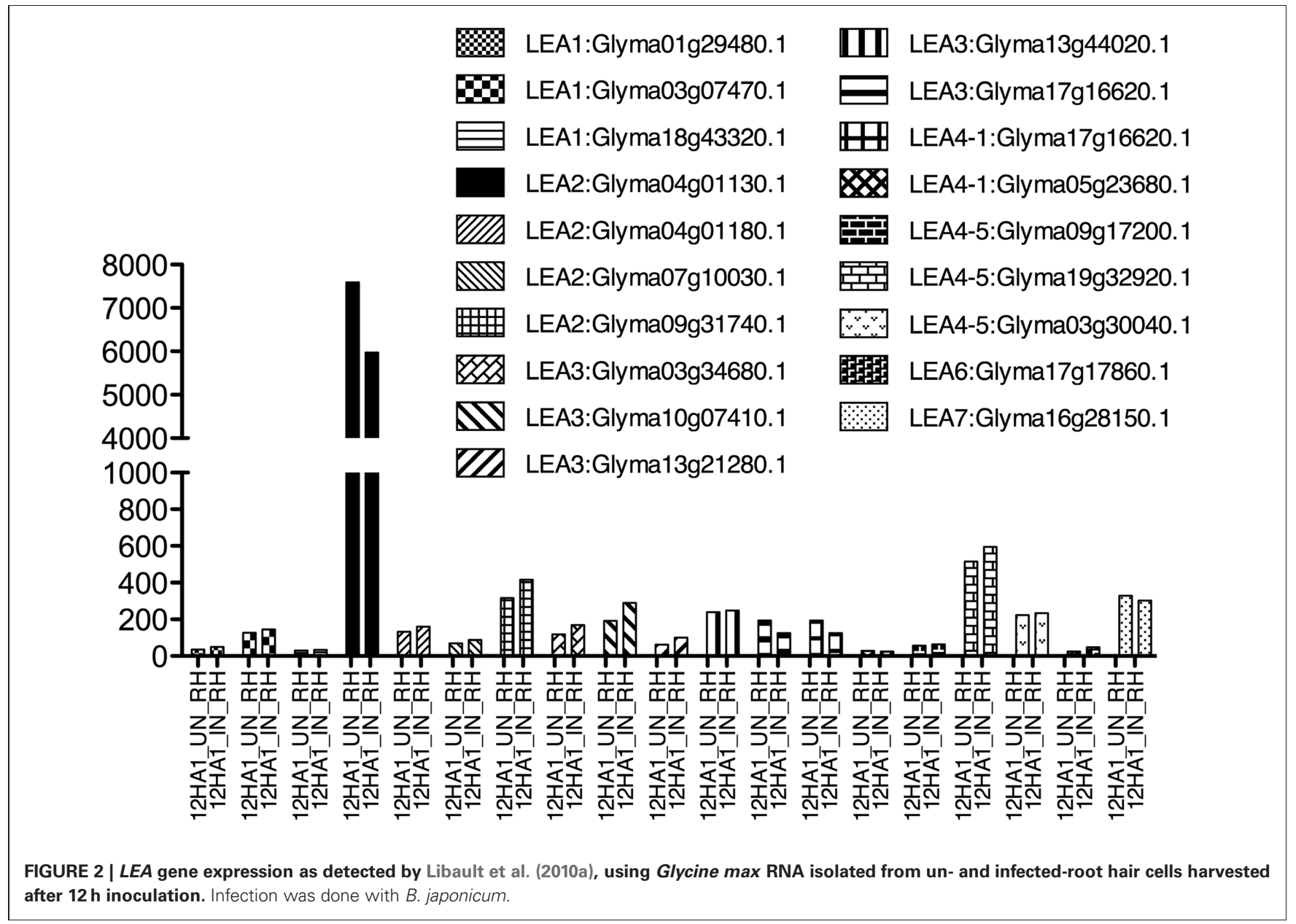


sensitivity of detection and protein extraction methods, and the properties of the proteins.

\section{ACCUMULATION IN NODULES}

Nitrogen fixation is one of the fundamental processes in nature, carried out by $\mathrm{N}_{2}$ fixing-organisms. In some cases, this activity is possible because the ability of some bacteria to establish symbiosis with legumes, a process that implies a perfect coordination between the plant and the bacteria and that results in the formation of a new organ, the nodule, where nitrogen fixation occurs. Rhizobium is a bacterial group that by means of a fine molecular communication detects, recognizes and establishes symbiosis with plants. In the nodule, rhizobia develop as bacteroids that will be the $\mathrm{N}_{2}$ fixation competent bacterial stage (Oldroyd and Downie, 2008). Because the relevance of nodules in this function, the accumulation of LEA proteins and their transcripts was investigated in these organs from the M. truncatula Gene Expression Atlas (MtGEA) Project (Benedito et al., 2008) and G. max RNA-Seq atlas (Libault et al., 2010b; Severin et al., 2010), where some expression patterns are available. The results showed that LEA genes are expressed in nodules; however, we did not find representatives for all groups (Figure 3). LEA1 and LEA3 transcripts were not detected in these two legumes. The identified LEA transcripts corresponded to group 2 (Medtr7g086340.1, Medtr3g117290.1, and Glyma04g01130), group 4 (only one from M. truncatula, Medtr7g093140.1- LEA4-5 in 4 dpi nodule primordia), group 6 (G. max LEA6 genes), and group 7 (only one gene from G. max, Glyma16g28150.1).

We found differences between the expression of $L E A$ genes in M. truncatula and G. max and also between the different groups of LEA proteins (see Figure 3). LEA2 transcripts were detected in both species for genes Medtr7g086340.1, Medtr3g117290.1, and Glyma04g01130. Only one LEA4 protein was detected in primordia (4 days after inoculation) of M. truncatula (Medtr7g093140.1LEA4-5), while all LEA6 genes from G. max were detected in nodules. From group 7 only one gene (Glyma16g28150.1) was found. A significant increase in the accumulation of two group 2 LEA protein transcripts (GmLEA8 and GmLEA10) has also been detected in soybean roots after 35 days of inoculation with Bradyrhizobium japonicum in well-watered plants; unexpectedly, lower accumulation levels of both transcripts were found in drought-stressed plants after the same inoculation time, suggesting that these LEA proteins may play a role during B. japonicum infection, induced by the "stress" caused during this process and/or by some developmental cue. The accumulation patterns for these transcripts have been also obtained from the corresponding nodules, where transcripts were not detected in well-irrigated plants, while nodules from drought-stressed plants, as expected, showed increased accumulation levels for both LEA mRNAs (Porcel et al., 2005). The response of these two genes (GmLEA8 and GmLEA10) was also examined in soybean plants colonized by the arbuscular mycorrhizal fungus Glomus mosseae, grown under optimal irrigation or stress conditions. Even though, roots of inoculated drought-stressed plants showed high accumulation of these transcripts, the accumulation levels were lower than non-inoculated stressed plants, supporting the idea that the association with mycorrhiza brings on a plant tolerance response possibly due to changes in root morphology produced by this symbiosis. Accordingly, no expression was detected upon mycorrhiza inoculation in contrast to the accumulation response to B. japonicum infection (Porcel et al., 2005).

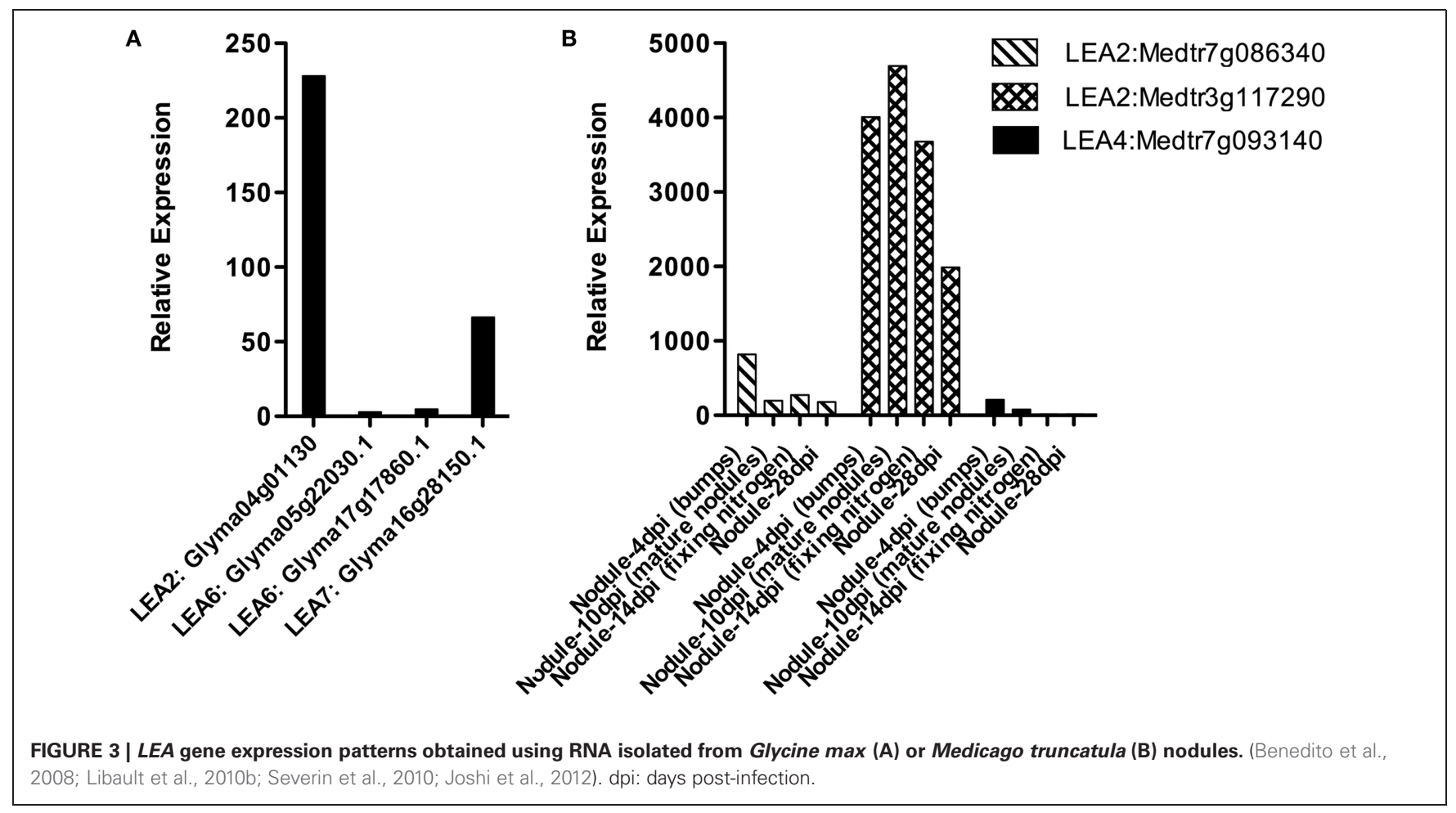




\section{CONCLUDING REMARKS}

LEA proteins have represented for a long time an enigma in plant biology. The lack of similarity to other proteins of known function and their high structural flexibility has hampered the progress regarding their activity and the implicated mechanisms. However, the high association between their accumulation and different levels of water deficit, caused during development or by the environment, has contributed to build hypotheses regarding their role in the plant tolerance to those conditions that produce low water availability, as drought, salinity, or extreme temperatures.

This has led to relevant advances concerning their transcript and protein accumulation and expression patterns in response to abiotic stress or throughout development, their participation in these processes, and their structural properties. The available information presents LEA proteins as a set of proteins whose participation in plant tolerance to water deficit conditions has gained experimental support, and also as proteins whose flexible structure turns them as a good model to find the functional relevance of this flexibility in the plant response to stress.

Because most of the information regarding LEA proteins has been obtained from Arabidopsis, in this work we have focused on relevant data reported from legumes, to explore whether the observations made in Arabidopsis can be extended to this family of plants, or whether legume LEA proteins present some distinctive structural or functional features.

The analysis of the compiled data from literature and from the available databases showed that most of the structural characteristics recognized in these proteins are conserved in their corresponding homologs in the different legume LEA protein groups; however, it also revealed an additional motif in LEA2 proteins not detected to date in other plant families. It is worthwhile mentioning that LEA7 proteins, a group not present in Arabidopsis but detected in many other species, is also in legumes. As expected, the transcript and protein accumulation patterns available show their responsiveness to water deficit imposed by salinity, high osmolarity, dehydration, and low temperatures, supporting their participation in legume responses to these stressful conditions.

\section{REFERENCES}

Aghaei, K., Ehsanpour, A. A., Shah, A. H., and Komatsu, S. (2009). Proteome analysis of soybean hypocotyl and root under salt stress. Amino Acids 36, 91-98. doi: 10.1016/j.bbamem.2010.09.010

Bai, Y., Yang, Q., Kang, J., Sun, Y., Gruber, M., and Chao, Y. (2012). Isolation and functional characterization of a Medicago sativa L. gene, MsLEA. Mol. Biol. Rep. 39, 2883-2892. doi: 10.1016/j.bbrc.2011.11.067

Bailey, T. L., Boden, M., Buske, F. A., Frith, M., Grant, C. E., Clementi, L., et al. (2009). MEME SUITE: tools for motif discovery and searching. Nucleic Acid Res. 37, W202-W208. doi: 10.1104/pp.120. 1.237

Of particular interest was the finding that various LEA transcripts from all groups accumulate in meristematic regions, even in plants grown under optimal growth conditions supporting a protective role in these fragile but critical regions for plant adaptation and survival, as proposed by Olvera-Carrillo et al. (2010), given that the over-expression or deficiency of a LEA4 protein led to an increase or a reduction, respectively, in the number of floral and axillar buds in Arabidopsis plants subjected to water deficit treatments. Moreover, different LEA transcripts were also detected in root hairs, which as in the previous case are frail but essential organs not only for water absorption but also to establish symbiosis between legumes and rhizobia needed for nitrogen fixation. Nodules also contain some LEA proteins; however, not all LEA protein groups seem to be present in the different types of nodules. A more detailed analysis is required, where the transcript levels of LEA protein from different groups is determined considering different stages during nodule development. With this limited and heterogeneous information it is difficult to be certain of the absence of some LEA protein groups in nodules. Similarly, at this point proposing tentative roles of these proteins during nodule development under optimal conditions would be highly speculative.

The information in this work regarding LEA proteins in legumes exposes the relevance of these proteins in the response and adaptation of this family of plants to water limiting environments, as it has been shown in other species, and justifies further studies to understand their function and participation in the plant responses to stress and during development. Also, it opens new interesting avenues regarding their role in meristematic regions and other fragile organs, such as root hairs, of particular importance in legumes symbiosis. All this predicts an appealing scenario full of surprises and knowledge.

\section{ACKNOWLEDGMENTS}

We are grateful to J. L. Reyes for discussions and critical review and to CONACyT-Mexico (132258) and DGAPA-PAPIIT-UNAM (IN-208212) for generous financial support.

profiling diversity of the LEA (Late Embryogenesis Abundant) protein gene family in Arabidopsis thaliana. Plant Mol. Biol. 67, 107-124. doi: 10.1016/j.bbrc.2006.07.003

Black, M., Corbineau, F., Gee, H., and Come, D. (1999). Water content, raffinose, and dehydrins in the induction of desiccation tolerance in immature wheat embryos. Plant Physiol. 120, 463-472. doi: 10.1104/pp.011171

Blackman, S. A., Wettlaufer, S. H., Obendorf, R. L., and Leopold, A. C. (1991). Maturation proteins associated with desiccation tolerance in soybean. Plant Physiol. 96, 868-874. doi: 10.1104/pp.109.136697

Boudet, J., Buitink, J., Hoekstra, F. A., Rogniaux, H., Larré, C., Satour, P., et al. (2006). Comparative analysis of the heat stable proteome of radicles of Medicago truncatula seeds during germination identifies late embryogenesis abundant proteins associated with desiccation tolerance. Plant Physiol. 140, 1418-1436. doi: 10.1007/s10535007-0133-6

Browne, J. A., Dolan, K. M., Tyson, T., Goyal, K., Tunnacliffe, A., and Burnell, A. M. (2004). Dehydrationspecific induction of hydrophilic protein genes in the anhydrobiotic nematode Aphelenchus avenae. Eukaryot. Cell 3, 966-975. doi: 10.1371/journal.pone.0049522

Campbell, S. A., and Close, T. J. (1997). Dehydrins: genes, proteins, and associations with phenotypic traits. New Phytol. 137, 61-74. doi: 10.1104/pp.109.148379 
Campos, F., Zamudio, F., and Covarrubias, A. A. (2006). Two different late embryogenesis abundant proteins from Arabidopsis thaliana contain specific domains that inhibit Escherichia coli growth. Biochem. Biophys. Res. Commun. $342,406-413$.

Cárdenas, L. (2009). New findings in the mechanisms regulating polar growth in root hair cells. Plant Signal. Behav. 4, 4-8.

Chakrabortee, S., Boschetti, C., Walton, L. J., Sarkar, S., Rubinsztein, D. C., and Tunnacliffe, A. (2007). Hydrophilic protein associated with desiccation tolerance exhibits broad protein stabilization function. Proc. Natl. Acad. Sci. U.S.A. 104, 18073-18078. doi: $10.1093 / \mathrm{pcp} / \mathrm{pcs} 040$

Chatelain, E., Hundertmark, M., Leprince, O., Gall, S. L., Satour, P., Deligny-Penninck, S., et al. (2012). Temporal profiling of the heat-stable proteome during late maturation of Medicago truncatula seeds identifies a restricted subset of late embryogenesis abundant proteins associated with longevity. Plant Cell Environ. 35, 1440-1455. doi: 10.1093/nar/15.8.3607

Cheng, J. C., Seeley, K. A., Goupil, P., and Sung, Z. R. (1996). Expression of DC8 is associated with, but not dependent on embryogenesis. Plant Mol. Biol. 31, 127-141.

Clark, M. S., Denekamp, N. Y., Thorne, M. A., Reinhardt, R., Drungowski, M., Albrecht, M. W., et al. (2012). Long-term survival of hydrated resting eggs from Brachionus plicatilis. PLoS ONE 7:e29365. doi: 10.1016/j.bbrc.2005.03.165

Colmenero-Flores, J. M., Campos, F., Garciarrubio, A., and Covarrubias, A. A. (1997). Characterization of Phaseolus vulgaris cDNA clones responsive to water deficit: identification of a novel late embryogenesis abundant-like protein. Plant Mol. Biol. 35, 393-405. doi: 10.1007/s00284-0099552-2

Colmenero-Flores, J. M., Moreno, L. P., Smith, C., and Covarrubias, A. A. (1999). Pvlea-18, a member of a new late-embryogenesis-abundant protein family that accumulates during water stress and in the growing regions of well-irrigated bean seedlings. Plant Physiol. 120, 93-103. doi: 10.1093/pcp/pcr052

Dominguez, P. G., Frankel, N., Mazuch, J., Balbo, I., Iusem, N., Fernie, A. R., et al. (2013). ASR1 mediates glucose-hormone crosstalk by affecting sugar trafficking in tobacco plants. Plant
Physiol. 161, 1486-1500. doi: $10.1093 / \mathrm{pcp} / \mathrm{pct} 047$

Duan, J., and Cai, W. (2012). OsLEA32 , an abiotic stress induced gene of rice plays a key role in salt and drought tolerante. PLOS ONE 7:e45117. doi: $10.1093 / \mathrm{pcp} / \mathrm{pcn} 185$

Dure, III L. (1993). "Structural motifs in Lea proteins," in Plant Responses to Cellular Dehydration During Environmental Stress, eds T. J. Close and E. A. Bray. (Rockville, MD: The American Society of Plant Physiologists), 91-103. doi: 10.1104/pp.105.072967

Dure, III L., Crouch, M., Harada, J., Ho, T. H. D., Mundy, J., Quatrano, R., et al. (1989). Common amino acid sequence domains among the LEA proteins of higher plants. Plant Mol. Biol. 12, 475-486. doi: 10.1016/j.bbrc.2006.11.115

Dure, III L., Greenway, S. C., and Galau, G. A. (1981). Developmental biochemistry of cottonseed embryogenesis and germination: changing messenger ribonucleic acid populations as shown by in vitro and in vivo protein synthesis. Biochemistry 20, 4162-4168. doi: 10.1016/j.jinsphys.2013.01.004

Eriksson, S. K., and Harryson, P. (2011). "Dehydrins: molecular biology, structure and function," in Plant Desiccation Tolerance, eds U. Lüttge, B. Beck, and D Bartels (Berlin-Heidelberg: $\quad$ SpringerVerlag), 289-305. doi: 10.1271/bbb. 67.1832

Gai, Y. P., Ji, X. L., Lu, W., Han, X. J., Yang, G. D., and Zheng, C. C. (2011). A novel late embryogenesis abundant like protein associated with chilling stress in Nicotiana tabacum cv. bright yellow-2 cell suspension culture. Mol. Cel. Proteom. 10:M111.010363. doi: 10.1023/A:1010607223533

Garay-Arroyo, A., Colmenero-Flores, J. M., Garciarrubio, A., and Covarrubias, A. A. (2000). Highly hydrophilic proteins in prokaryotes and eukaryotes are common during conditions of water deficit. J. Biol. Chem. 275, 5668-5674. doi: 10.1104/pp.001925

Gepts, P., Beavis, W. D., Brummer, S., Shoemaker, R. C., Stalker, H. T., Weeden, N. F., et al. (2005). Legumes as a model plant family. genomics for food and feed report of the crosslegume advances through genomics conference. Plant Physiol. 137, 1228-1235. doi: 10.1146/annurev. arplant.59.032607.092839

Goday, A., Jensen, A. B., CulianezMacia, F. A., Mar Alba, M., Figueras, M., Serratosa, J., et al.
(1994). The maize abscisic acid-responsive protein Rab17 is located in the nucleus and interacts with nuclear localization signals. Plant Cell 6, 351-360. doi: 10.1104/pp.110.158964

Goormachtig, S., Capoen, W., and Holsters, M. (2004). Rhizobium infection: lessons from the versatile nodulation behaviour of watertolerant legumes. Trends Plant Sci. 9 , 518-522.

Goyal, K., Pinelli, C., Maslen, S. L., Rastogi, R. K., Stephens, E. and Tunnacliffe, A. (2005a). Dehydration-regulated processing of late embryogenesis abundant protein in a desiccationtolerant nematode. FEBS Lett. 579, 4093-4098. doi: 10.1016/j.bbamem.2011.03.009

Goyal, K., Walton, L. J., and Tunnacliffe, A. (2005b). LEA proteins prevent protein aggregation due to water stress. Biochem. J. 388, 151-157. doi: 10.1093/jxb/eri188

Grelet, J., Benamar, A., Teyssier, E., Avelange-Macherel, M. H., Grunwald, D., and Macherel, D. (2005). Identification in pea seed mitochondria of a lateembryogenesis abundant protein able to protect enzymes from drying. Plant Physiol. 137, 157-167. doi: 10.1007/BF00201336

Hand, S. C., Menze, M. A., Toner, M., Boswell, L., and Moore, D. (2011). LEA proteins during water stress: not just for plants anymore. Annu. Rev. Physiol. 73, 115-134. doi: $\quad 10.1111 / j .1365-3040.2008$ 01879.x

Hanin, M., Brini, F., Ebel, C. H., Toda, Y., Takeda, S., and Masmoudi, K. (2011). Plant dehydrins and stress tolerance. versatile proteins for complex mechanisms. Plant Signal. Behav. 6, 1503-1509. doi: 10.1111/j.1365-3040.2005.01317.x

Hara, M. (2010). The multifunctionality of dehydrins. an overview. Plant Signal. Behav. 5, 503-508. doi 10.1186/1471-2229-10-160

Hara, M., Fujinaga, M., and Kuboi, T. (2005). Metal binding by citrus dehydrin with histidine-rich domains. J. Exp. Bot. 56, 2695-2703. doi: 10.2478/s11658-006-0044-0

Hara, M., Shinoda, Y., Tanaka, Y., and Kuboi, T. (2009). DNA binding of citrus dehydrin promoted by zinc ion. Plant Cell Environ. 32, 532-541. doi: 10.1111/j.1365313X.2005.02603.x

Hara, M., Wakasugi, Y., Ikoma, Y., Yano, M., Ogawa, K., and Kuboi, T. (1999). cDNA sequence and expression of a cold-responsive gene in Citrus unshiu. Biosci.
Biotechnol. Biochem. 63, 433-437. doi: 10.1073/pnas.0306154101

Honjoh, K., Yoshimoto, M., Joh, T., Kajiwara, T., Miyamoto, T., and Hatano, S. (1995). Isolation and characterization of hardeninginduced proteins in Chlorella vulgaris C-27: identification of late embryogenesis abundant proteins. Plant Cell Physiol. 36, 1421-1430. doi: 10.1007/s00497-006-0035-3

Houde, M., Daniel, C., Lachapelle, M., Allard, F., Laliberte, S., and Sarhan, F. (1995). Immunolocalization of freezing-tolerance-associated proteins in the cytoplasm and nucleoplasm of wheat crown tissues. Plant J. 8, 583-593. doi: 10.1016/j.plantsci.2012.07.012

Hsing, Y. C., Chen, Z. Y., Shih, M. D., Hsieh, J. S., and Chow, T. Y. (1995). Unusual sequences of group 3 LEA mRNA inducible by maturation or drying in soybean seeds. Plant Mol. Biol. 29, 863-868. doi: $10.1093 / \mathrm{pcp} / \mathrm{pcq} 005$

Hundertmark, M., Dimova, R. Lengefeld, J., Seckler, R., and Hincha, D. K. (2011). The intrinsically disordered late embryogenesis abundant protein LEA18 from Arabidopsis thaliana modulates membrane stability through binding and folding. Biochim. Biophys. Acta-Biomem. 1808, 446-453. doi: 10.1104/pp.015891

Hundertmark, M., and Hincha, D. K. (2008). LEA (late embryogenesis abundant) proteins and their encoding genes in Arabidopsis thaliana. BMC Genom. 9:118. doi: 10.1007/s11103-004-4680-3

Hundertmark, M., Popova, A. V., Rausch, S., Seckler, R., and Hincha, D. K. (2012). Influence of drying on the secondary structure of intrinsically disordered and globular proteins. Biochem. Biophy. Res. Com. 417, 122-128. doi: 10.1104/pp.010521

Ismail, A. M., Hall, A. E., and Close, T. J. (1999). Purification and partial characterization of a dehydrin involved in chilling tolerance during seedling emergence of cowpea. Plant Physiol. 120, 237-244. doi: 10.1007/s004250050424

Joh, T., Honjoh, K., Yoshimoto, M., Funabashi, J., Miyamoto, T., and Hatano, S. (1995). Molecular cloning and expression of hardening-induced genes in Chlorella vulgaris C-27: the most abundant clone encodes a late embryogenesis abundant protein. Plant Cell Physiol. 36, 85-93. doi: 10.1007/s12038-011-9058-5

Joshi, T., Patil, K., Fitzpatrick, M. R., Franklin, L. D., Yao, Q., Cook, J. R., 
et al. (2012). Soybean Knowledge Base (SoyKB): a web resource for soybean translational genomics. BMC Genom. 13(Suppl. 1):S15. doi: 10.1016/j.bbamem.2010.05.015

Kalaiselvi, K., and Manickam, A. (1999). Characterization of an axis-specific protein from mung bean expressed late during seed maturation. Plant Physiol. Biochem. 37, 203-208. doi: 10.1016/j.bbamem.2010.06.029

Kikawada, T., Nakahara, Y., Kanamori, Y., Iwata, K.-i., Watanabe, M., and McGee, B., et al. (2006). Dehydration-induced expression of LEA proteins in an anhydrobiotic chironomid. Biochem. Biophys. Res. Com. 348, 56-61.

Koag, M. C., Fenton, R. D., Wilkens, S., and Close, T. J. (2003). The binding of maize DHN1 to lipid vesicles. Gain of structure and lipid specificity. Plant Physiol. 131, 309-316.

Koag, M. C., Wilkens, S., Fenton, R. D., Resnik, J., Vo, E., and Close, T. J. (2009). The K-segment of maize DHN1 mediates binding to anionic phospholipid vesicles and concomitant structural changes. Plant Physiol. 150, 1503-1514. doi: 10.1093/jexbot/51.348.1211

Kosová, K., Vítámvás, P., and Prášil, I. T. (2007). The role of dehydrins in plant response to cold. Biol. Plant. 51, 601-617. doi: 10.1002/pmic.200700259

Le, D. T., Nishiyama, R., Watanabe, Y., Tanaka, M., Seki, M., Ham le, H., et al. (2012). Differential gene expression in soybean leaf tissues at late developmental stages under drought stress revealed by genomewide transcriptome analysis. PLoS ONE 7:e49522. doi: 10.1371/journal.pone.0049522

Libault, M., Farmer, A., Brechenmacher, L., Drnevich, J., Langley, R. J., Bilgin, D. D., et al. (2010a). Complete transcriptome of the soybean root hair cell, a single-cell model, and its alteration in response to Bradyrhizobium japonicum infection. Plant Physiol. 152, 541-552. doi: 10.1016/S0167-4838(00)00220-X

Libault, M., Farmer, A., Joshi, T., Takahashi, K., Langley, R. J., Franklin, L. D., et al. (2010b). An integrated transcriptome atlas of the crop model Glycine max, and its use in comparative analyses in plants. Plant J. 63, 86-99. doi: 10.1021/pr060336t

Lisse, T., Bartels, D., Kalbitzer, H. R., and Jaenicke, R. (1996). The recombinant dehydrin-like desiccation stress protein from the resurrection plant Craterostigma plant- agineum displays no defined threedimensional structure in its native state. Biol. Chem. 377, 555-561.

Lin, C. H., Peng, P. H., Ko, C. Y., Markhart, A. H., and Lin, T. Y. (2012). Characterization of a novel Y2K-type dehydrin VrDhn1 from Vigna radiata. Plant Cell Physiol. 53, 930-942.

Litts, J. C., Colwell, G. W., Chakerian, R. L., and Quatrano, R. S. (1987). The nucleotide sequence of a cDNA clone encoding the wheat Em protein. Nucleic Acid Res. 15 3607-3618.

Litts, J. C., Colwell, G. W., Chakerian, R. L., and Quatrano, R. S. (1991). Sequence analysis of a functional member of the Em gene family from wheat. DNA Seq. 1, 263-274.

Liu, G., Xu, H., Zhang, L., and Zheng, Y. (2011). Fe binding properties of two soybean (Glycine max L.) LEA4 proteins associated with antioxidant activity. Plant Cell Physiol. 52, 994-1002.

Liu, Y., Wang, L., Sun, L., Pan, J., Kong, X., Zhang, M., et al. (2013). ZmLEA3, a multifunctional group 3 LEA protein from maize Zea mays $\mathrm{L}$, is involved in biotic and abiotic stresses. Plant Cell Physiol. 54, 944-959. doi: 10.1093/pcp/pct047

Liu, Y., and Zheng, Y. (2005). PM2, a group 3 LEA protein from soybean, and its 22-mer repeating region confer salt tolerance in Escherichia coli. Biochem. Biophys. Res. Com. 331, 325-332.

Liu, Y., Zheng, Y., Zhang, Y., Wang, W., and Li, R. (2010). Soybean PM2 protein (LEA3) confers the tolerance of Escherichia coli and stabilization of enzyme activity under diverse stresses. Curr. Microbiol. 60, 373-378.

Manfre, A. J., LaHatte, G. A., Climer, C. R., and Marcotte, W. R. Jr. (2009). Seed dehydration and the establishment of desiccation tolerance during seed maturation is altered in the Arabidopsis thaliana mutant atem61. Plant Cell Physiol. 50, 243-253.

Manfre, A. J., Lanni, L. M., and Marcotte, W. R. Jr. (2006). The Arabidopsis group 1 LATE EMBRYOGENESIS ABUNDANT protein ATEM6 is required for normal seed development. Plant Physiol. 140, 140-149.

Maskin, L., Frankel, N., Gudesblat, G., Demergasso, M. J., Pietrasanta, L. I., and Iusem, N. D. (2007). Dimerization and DNA-binding of ASR1, a small hydrophilic protein abundant in plant tissues suffering from water loss. Biochem. Biophys. Res. Com. 352, 831-835.
Marunde, M. R., Samarajeewa, D. A., Anderson, J., Li, S., Hand, S. C., and Menze, M. A. (2013). Improved tolerance to salt and water stress in Drosophila melanogaster cells conferred by late embryogenesis abundant protein. J. Insect Physiol. 59, 377-386.

Momma, M., Kaneko, S., Haraguchi, K., and Matsukura, U. (2003) Peptide mapping and assessment of cryoprotective activity of $26 / 27-\mathrm{kDa}$ dehydrin from soybean seeds. Biosci. Biotechnol. Biochem. 67, 1832-1835.

Moreno-Fonseca, L. P., and Covarrubias, A. A. (2001). Downstream DNA sequences are required to modulate Pvlea-18 gene expression in response to dehydration. Plant Mol. Biol. 45, 501-515.

Ndong, C., Danyluk, J., Wilson, K. E. Pocock, T., Huner, N. P. A., and Sarhan, F. (2002). Cold-regulated cereal chloroplast late embryogenesis abundant-like proteins. molecular characterization and functional analyses. Plant Physiol. 129, 1368-1381.

Oldroyd, G. E., and Downie, J. A (2008). Coordinating nodule morphogenesis with rhizobial infection in legumes. Annu. Rev. Plant Biol. 59, 519-546.

Olvera-Carrillo, Y., Campos, F. Reyes, J. L., Garciarrubio, A., and Covarrubias, A. A. (2010). Functional analysis of the group 4 late embryogenesis abundant proteins reveals their relevance in the adaptive response during water deficit in Arabidopsis. Plant Physiol. 154, 373-390.

Olvera-Carrillo, Y., Reyes, J. L., and Covarrubias, A. A. (2011). Late Embryogenesis Abundant proteins: versatile players in the plant adaptation to water limiting environments. Plant Signal. Behav. 64, 1-4.

Popova, A. V., Hundertmark, M. Seckler, R., and Hincha, D. K. (2011). Structural transitions in the intrinsically disordered plant dehydration stress protein LEA7 upon drying are modulated by the presence of membranes. Biochim. Biophys. Acta 1808, 1879-1887.

Porcel, R., Azcón, R., and RuizLozano, J. D. (2005). Evaluation of the role of genes encoding for dehydrin proteins (LEA D-11) during drought stress in arbuscular mycorrhizal Glycine max and Lactuca sativa plants. J. Exp. Bot. 56, 1933-1942.

Puhakainen, T., Hess, M. W., Makela, P., Svensson, J., Heino, P., and Palva, E. T. (2004). Overexpression of multiple dehydrin genes enhances tolerance to freezing stress in
Arabidopsis. Plant Mol. Biol. 54, 743-753.

Raghavan, V., and Kamalay, J. C. (1993). Expression of two cloned mRNA sequences during development and germination of spores of the sensitive fern, Onoclea sensibilis L. Planta 189, 1-9.

Reyes, J. L., Campos, F., Wei, H., Arora, R., Yang, Y., Karlson, D. T., et al. (2008). Functional dissection of hydrophilins during in vitro freeze protection. Plant Cell Environ. 31, 1781-1790.

Reyes, J. L., Rodrigo, M. J., ColmeneroFlores, J. M., Gil, J. V., GarayArroyo, A., Campos, F., et al. (2005). Hydrophilins from distant organisms can protect enzymatic activities from water limitation effects in vitro. Plant Cell Environ. 28, 709-718.

Riera, M., Figueras, M., López, C., Goday, A., and Pages, M. (2004). Protein kinase CK2 modulates developmental functions of the abscisic acid responsive protein Rab17 from maize. Proc. Natl. Acad. Sci. U.S.A. 101, 9879-9884.

Rorat, T. (2006). Plant dehydrins Tissue localization, structure and function. Cell Mol. Biol. Lett. 11, 536-556.

Saavedra, L., Svensson, J., Carballo, V., Izmendi, D., Welin, B., and Vidal, S. (2006). A dehydrin gene in Physcomitrella patens is required for salt and osmotic stress tolerance. Plant J. 45, 237-249.

Severin, A. J., Woody, J. L., Bolon, Y. T. Joseph, B., Diers, B. W., Farmer, A. D., et al. (2010). RNA-Seq Atlas of Glycine max: a guide to the soybean transcriptome. BMC Plant Biol. 10:160. doi: 10.1186/1471-2229-10160

Sheoran, I. S., Sproule, K. A., Olson, D. J. H., Ross, A. R. S., and Sawhney, V. K. (2006). Proteome profile and functional classification of proteins in Arabidopsis thaliana (Landsberg erecta) mature pollen. Sex. Plant Reprod. 19, 185-196.

Shih, M. D., Hsieh, T. Y., Jian, W. T. Wu, M. T., Yang, S. J., Hoekstra, F. A., et al. (2012). Functional studies of soybean (Glycine max L.) seed LEA proteins GmPM6, GmPM11, and GmPM30 by CD and FTIR spectroscopy. Plant Sci. 196, 152-159.

Shih, M. D., Hsieh, T. Y., Lin, T. P., Hsing, Y. I. C., and Hoekstra, F. A. (2010). Characterization of two soybean (Glycine $\max$ L.) LEA IV proteins by circular dichroism and Fourier transform infrared spectrometry. Plant Cell Physiol. 51, 395-407. 
Shih, M. D., Lin, S. C., Hsieh, J. S., Tsou, C. H., Chow, T. Y., Lin, T. P., et al. (2004). Gene cloning and characterization of a soybean (Glycine max L.) LEA protein, GmPM16. Plant Mol. Biol. 56, 689-703.

Soulages, J. L., Kim, K., Arrese, E. L., Walters, C., and Cushman, J. C. (2003). Conformation of a group 2 late embryogenesis abundant protein from soybean. evidence of poly (L-proline)-type II structure. Plant Physiol. 131, 963-975.

Soulages, J. L., Kim, K., Walters, C., and Cushman, J. C. (2002). Temperature-induced extended helix/random coil transitions in a group 1 late embryogenesisabundant protein from soybean. Plant Physiol. 128, 822-832.

Stacy, R. A. P., and Aalen, R. B. (1998). Identification of sequence homology between the internal hydrophilic repeated motifs of Group 1 late-embryogenesisabundant proteins in plants and hydrophilic repeats of the general stress protein GsiB of Bacillus subtilis. Planta 206, 476-478.

Su, L., Zhao, C. Z., Bi, Y. P., Wan, S. B., Xia, H., and Wang, X. J.
(2011). Isolation and expression analysis of $L E A$ genes in peanut (Arachis hypogaea L.). J. Biosci. 36, 223-228.

Thalhammer, A., Hundertmark, M., Popova, A. V., Seckler, R., and Hincha, D. K. (2010). Interaction of two intrinsically disordered plant stress proteins (COR15A and COR15B) with lipid membranes in the dry state. Biochim. Biophys. Acta 1798, 1812-1820.

Tolleter, D., Hincha, D. K., and Macherel, D. (2010). A mitochondrial late embryogenesis abundant protein stabilizes model membranes in the dry state. Biochim. Biophys. Acta 1798, 1926-1933.

Varshney, R. K., Chen, W., Li, Y., Bharti, A. K., Saxena, R. K., Schlueter, J. A., et al. (2012). Draft genome sequence of pigeonpea (Cajanus cajan), an orphan legume crop of resource-poor farmers. Nat. Biotechnol. 30, Aì83-Aì89.

Varshney, R. K., Song, C., Saxena, R. K., Azam, S., Yu, S., Sharpe, A. G., et al. (2013). Draft genome sequence of chickpea (Cicer arietinum) provides a resource for trait improvement. Nat. Biotechnol. 31, Aì240-Aì246.

Vicient, C. M., Hull, G., Guilleminot, J., Devic, M., and Delseny, M. (2000). Differential expression of the Arabidopsis genes coding for Em-like proteins. J. Exp. Bot. 51, 1211-1220.

Wang, W., Meng, B., Chen, W., Ge, X., Liu, S., and Yu, J. (2007). A proteomic study on postdiapaused embryonic development of brine shrimp (Artemia franciscana). Proteomics 7, 3580-3591.

Whitsitt, M. S., Collins, R. G., and Mullet, J. E. (1997). Modulation of dehydration tolerance in soybean seedlings (Dehydrin Matl is induced by dehydration but not by abscisic acid). Plant Physiol. 114, 917-925.

Wolkers, W. F., McCready, S., Brandt, W. F., Lindsey, G. G., and Hoekstra, F. A. (2001). Isolation and characterization of a D-7 LEA protein from pollen that stabilizes glasses in vitro. Biochim. Biophys. Acta 1544, 196-206.

Zhang, K., McKinlay, C., Hocart, C. H., and Djordjevic, M. A. (2006). The Medicago truncatula small protein proteome and peptidome. J. Proteome Res. 5, 3355-3367.

Conflict of Interest Statement: The authors declare that the research was conducted in the absence of any commercial or financial relationships that could be construed as a potential conflict of interest.

Received: 07 February 2013; accepted: 23 May 2013; published online: 25 June 2013.

Citation: Battaglia $M$ and Covarrubias AA (2013) Late Embryogenesis Abundant (LEA) proteins in legumes. Front. Plant Sci. 4:190. doi: 10.3389/fpls. 2013.00190

This article was submitted to Frontiers in Plant Genetics and Genomics, a specialty of Frontiers in Plant Science.

Copyright (c) 2013 Battaglia and Covarrubias. This is an open-access article distributed under the terms of the Creative Commons Attribution License, which permits use, distribution and reproduction in other forums, provided the original authors and source are credited and subject to any copyright notices concerning any third-party graphics etc. 